\title{
Optimal Transmission Switching
}

\author{
Emily Bartholomew Fisher, Student Member, IEEE, Richard P. O’Neill, Michael C. Ferris
}

\begin{abstract}
In this paper, we formulate the problem of finding an optimal generation dispatch and transmission topology to meet a specific inflexible load as a mixed integer program. Our model is a mixed-integer linear program because it employs binary variables to represent the state of the equipment and linear relationships to describe the physical system. We find that on the standard 118-bus IEEE test case a savings of 25 percent in system dispatch cost can be achieved.
\end{abstract}

Index Terms-Integer programming, Power generation dispatch, Power system economics, Power transmission control, Power transmission economics, Transmission switching

\section{NOMENClature}

Indices

$n, m$ : nodes

$k$ : lines

$g$ : generators

$d$ : loads

$j$ : number of lines allowed to open in the generalized upper bound constraint

Variables

$\theta_{\mathrm{n}}$ : voltage angle at node $n$

$P_{n k}, P_{n g}, P_{n d}$ : real power flow to or from line $k$, generator $g$, or load $d$ to node $n$.

$z_{k}$ : binary variable indicating whether transmission line $k$ is removed from the system (open, $z_{k}=0$ ), or in the system (closed, $z_{k}=1$ )

$T C_{j}$ : Total system cost with $j$ lines

\section{Parameters}

$P_{n k}{ }^{\max }, P_{n k}{ }^{\min }$ : maximum and minimum capacity of line $k$

$P_{n g}^{\max }, P_{n g}{ }^{\text {min }}$ : maximum and minimum capacity of generator $g$

$\theta_{\mathrm{n}}{ }^{\max }, \theta_{\mathrm{n}}{ }^{\text {min }}$ : maximum and minimum voltage angle at node $n$

$\mathrm{c}_{\mathrm{ng}}$ : cost of generating electricity from generator $g$ located at node $n$

$B_{k}$ : electrical susceptance of line $k$

$M$ : large number

$C$ : upper limit on number of open transmission lines

Manuscript received July 2, 2007. This work was supported in part by the National Science Foundation under Grants DMS-0427689 and IIS-0511905 and the Air Force Office of Scientific Research Grant FA9550-07-1-0389.

E. B. Fisher is with Federal Energy Regulatory Commission (FERC), Washington, DC, USA, and the Johns Hopkins University, Baltimore, MD, USA (phone: 202-502-8093; e-mail: emily.bartholomew@ferc.gov).

R. P. O'Neill is the Chief Economic Advisor, FERC, (e-mail: richard.oneill@ferc.gov).

M. C. Ferris is a Professor at the University of Wisconsin, Madison, WI, USA (e-mail: ferris@cs.wisc.edu).
Sets

$\Lambda$ : Set of all transmission lines

$L$ : Set of open transmission lines in solution to transmission switching in which there is no upper limit on the number of open lines

$L_{j}$ : Set of open transmission lines in solution to transmission switching in which upper limit on open lines is $j$

\section{INTRODUCTION}

Tn large electric networks, transmission is traditionally characterized as a static system. In regulated areas, the utility dispatches generators over this fixed system to minimize cost, and in restructured regions generators use this network as a means to compete with one another. However, it is acknowledged, both formally and informally, that system operators can, and do, change the topology of systems to improve voltage profiles or increase capacity of a flowgate. ${ }^{1}$ In this paper we explore the implications of automating this practice, and meeting demand efficiently by optimizing the network through control of transmission line circuit breakers in addition to generator output. One reason this concept is particularly relevant now is the extreme difficulty in building transmission to meet growing demand; we propose one way to address this problem is to make more efficient use of the existing network.

Kirchhoff's laws allow the opening of lines to improve dispatch cost. Opening lines to improve dispatch is already being done to a small degree. Anecdotal evidence exists that some system operators switch lines in and out because of reactive power consumption or production of lines, or other reasons. ${ }^{2}$ The Northeast Power Coordinating Council includes "switch out internal transmission lines" in the list of possible actions to avoid abnormal voltage conditions [1] [2]. In addition, system operators have procedures in place to close lines quickly in case of emergency. In PJM, these Special Protection Schemes (SPSs) allow the operator to disconnect a line during normal operations but return it to service during a contingency.

Electric transmission planning has long been an important field in power system engineering, and many planning techniques have been developed and studied [3] [4] [5]. By necessity, these techniques focus on long-term decisions about investments and uncertainty, not operational decisions based on short-term system conditions. In this paper, we examine the possibilities of improved system dispatch by altering existing networks, which is a different problem from planning new lines or designing new networks. We assume the load is known with greater certainty, and the decisions are to change

\footnotetext{
${ }^{1}$ personal communication with Andy Ott, Vice President PJM.

2 personal communication with Steve Nauman, Vice President, Exelon.
} 
temporary network structure. The goal is not meeting probabilistic long-term reliability criteria, like LOLP or LOLE, or forecasted load growth, but to make each realization of the transmission topology optimal and reliable given the actual — or short-term forecasted - network conditions.

Transmission switching has been explored in the literature as a control method for problems such as over- or under-voltage situations, line overloads [6], loss and/or cost reduction [7], improving system security [8], or a combination of these [9] [10] [11]. Our investigation of optimal transmission switching extends this literature by probing the degree to which transmission switching could increase economic efficiency of power system dispatch. No other recent paper has explored the ability of transmission line switching to reduce dispatch cost, and of papers that have optimized cost none have used a mixed-integer formulation or presented the degree of savings possible [7].

The concept of optimal transmission switching for economic reasons was investigated by $\mathrm{O}^{\prime} \mathrm{Neill}$ et al. in a market context [12]. Here, the authors examined the dynamic operation and compensation of transmission lines (as well as phase angle regulators, FACTS devices, and other transmission technologies) on a small example network. In the current paper we restrict our analysis to transmission lines, and in particular apply optimal transmission switching to a well-known engineering test case to gain a better understanding of its potential impact in large and more realistic systems. The idea of market-based payments for transmission is outside the scope of this paper. In fact, the concepts presented here do not require the existence of a particular market design. All we assume is that the system operator or utility uses an optimal power flow technique to determine dispatch.

We formulate the problem as a mixed-integer linear program (MIP), based on the traditional DC optimal power flow (DCOPF) used to dispatch generators to meet load in an efficient manner. The integer variables are used to represent the state, closed or open, of each transmission line. Because of Kirchhoff's laws and power conservation, extending the DCOPF to include integer capability is not as straightforward as formulating a generator unit commitment problem. We then use this formulation to examine the potential for improving generation dispatches by optimizing transmission topology for a well known IEEE test system.

In proposing new operating techniques, it is important to keep in mind that the reliability of electricity networks is vital. Indeed, some may argue that optimizing transmission topology is unthinkable because of potential threats to reliability. In this paper we do not ignore the importance of reliability, nor are we suggesting switching transmission at the expense of reliable network operations. We are simply examining the potential for cost savings by streamlining and automating actions operators can currently take (such as SPSs) and improving network operation by making use of controllable components while maintaining system security. Lines that are open in the optimal dispatch of a network may be available to be switched back into the system as needed, as in PJM's SPSs. In cases where this may not be possible, optimal transmission switching can be conducted in conjunction with contingency analysis in order to maintain reliability levels while taking advantage of improved topology. However reliability is maintained, optimal transmission switching is not intrinsically incompatible with reliable operation of the grid.

The paper is organized as follows. Section III. presents the MIP formulation for the optimal transmission switching problem. Section IV. discusses results from using the new formulation on the well-known engineering test case. Section V. explores computational issues in solving this problem. Section VI. contains a brief discussion of practical implications of this method, and Section VII. concludes. The appendix explores heuristic methods that may aid in solving large MIPs, a topic important for eventual practical implementation.

\section{MIXED-INTEGER FormULATION}

As mentioned above, our formulation of the optimal transmission switching problem is based on the standard DCOPF. We use DC approximations [4] so the underlying problem is linear, but there is no reason an ACOPF could not be used instead other than the usual problems with computation time, convergence and proof of optimality in the nonlinear setting (see [13], [14] and [15] section 15.6).

\section{A. Basic Optimal Power Flow}

We start with a basic formulation of a DCOPF problem, as presented below. Generator costs (which are negative) are maximized, subject to physical constraints of the system and Kirchhoff's laws governing power flow. It is possible, and probably desirable from an implementation perspective, to include other costs in the objective function, such as generator start-up or cost associated with equipment switching or degradation. Making the objective function more robust would ensure all appropriate operating costs were being considered in the solution.

$$
T C=\operatorname{Max} \sum_{g} c_{n g} P_{n g}
$$

s.t.:

(1) $\theta_{n}^{\min } \leq \theta_{n} \leq \theta_{n}^{\max }$

$$
\begin{array}{ll}
P_{n g}^{\min } \leq P_{n g} \leq P_{n g}^{\max } & \text { for all } g \text { and } n \\
P_{n k}^{\min } \leq P_{n k} \leq P_{n k}^{\max } & \text { for all } k \text { and } n
\end{array}
$$

(4) $-\sum_{k} P_{n k}-\sum_{g} P_{n g}-\sum_{d} P_{n d}=0$ for all $n$ (5) $B_{k}\left(\theta_{n}-\theta_{m}\right)-P_{n k}=0 \quad$ for all lines $k$ with endpoints $n$ and $m$

The constraints represent physical operating limits of the 
network. Voltage angle limits are imposed by $(1) .{ }^{3}$ Constraint (2) limits the output of generator $g$ at node $n$ to its physical capabilities, and (3) limit the power flow across line $k$ at node $n^{4}$

Power balance at each node is enforced by (4), and Kirchhoff's laws are incorporated in constraint (5). $P_{n k}$ represents the power flow from line $k$ to node $n$, and therefore can be positive or negative depending on whether power is flowing into or out of the node. $P_{n g}$ and $P_{n d}$ represent the power injected by a generator or withdrawn by a load at node $n$, respectively. $P_{n g}$ is generally positive, whereas $P_{n d}$ is typically negative.

This formulation is very general, and could be expanded to include any other constraint or objective function that would represent the system more accurately.

\section{B. Optimal Power Flow with Optimal Transmission Switching}

Now we make changes to this basic formulation to allow for optimal transmission switching. In this new formulation, each line is assigned a binary variable, $z_{k}$, that represents whether the line is included in the system (the circuit breaker on that line is closed) or not (circuit breaker is open). Because of the peculiar characteristics of electricity, it is possible to improve the dispatch cost by removing a line from the network. Thus the optimization problem will either return the result that no lines are taken out with no change in the objective function value, or that one or more lines are opened with an improved objective function.

In the formation, the power flow of an open line must be constrained to zero; an open circuit or conductor can transmit no power. Because of the constraint representing Kirchhoff's laws, the formulation of the problem is more complicated than simply limiting the power flow to $P_{n k}{ }^{\max }$ or $P_{n k}{ }^{\min }$ times the binary variable. Whereas this formulation would allow no flow on an open line, it also limits power flow to zero on all lines that share terminal nodes with the open line, because the voltage angle difference is forced to zero. To account for this, the Kirchhoff's laws constraints (above, constraint 5) must be modified, as they are below (see constraints $5 \mathrm{a}$ and $5 \mathrm{~b}$ ).

$$
T C_{j}=\operatorname{Max} \sum_{k} C_{n k} P_{n k}
$$

s.t.:

$$
\begin{aligned}
& \theta_{n}^{\min } \leq \theta_{n} \leq \theta_{n}^{\max } \\
& P_{n g}^{\min } \leq P_{n g} \leq P_{n g}^{\max }
\end{aligned}
$$
for all $g$ and $n$

\footnotetext{
${ }^{3}$ Upper and lower voltage angle constraints were set to 0.6 and -0.6 radians, respectively. While these bounds are conservative, they aid in finding solutions in a timely manner. Loosening these bounds may result in improved solutions.
}

${ }^{4}$ For a DC model with no losses power flow does not have to be enforced at both ends of the lines; however if losses were modeled or a full AC power flow were used it would be important to model both ends of each line because real and reactive flows would be different at each end.
(3) $P_{n k}^{\min } Z_{k} \leq P_{n k} \leq P_{n k}^{\max } Z_{k} \quad$ for all $k$ at nodes $n$

(4) $-\sum_{k} P_{n k}-\sum_{g} P_{n g}-\sum_{d} P_{n d}=0$ for all $n$

(5a) $B_{k}\left(\theta_{n}-\theta_{m}\right)-P_{n k}+\left(1-z_{k}\right) M \geq 0$ for all lines $k$

with endpoints $n$ and $m$

(5b) $B_{k}\left(\theta_{n}-\theta_{m}\right)-P_{n k}-\left(1-z_{k}\right) M \leq 0$

(6) $\sum_{k}\left(1-z_{k}\right) \leq j$

Note that $M$ is a large number greater than or equal to $\mathrm{B}_{\mathrm{k}}\left(\theta_{\mathrm{n}}^{\max }-\theta_{\mathrm{m}}^{\min }\right)$, in the constraints (5a) and (5b). In this formulation we also added a generalized upper bound constraint (GUB) (constraint (6)) that can limit the number of open lines in the optimal network. This constraint is used to gain understanding about the effects of changing the network topology.

The following set notation will be used throughout the analysis section of the paper: The set of all lines is denoted as $\Lambda$. The subset of lines that are open in an optimal solution is denoted as $L_{j}$, where $j$ is the number of lines allowed to be open (i.e. constraint (6) in the above formulation would have $C=j$ ). Since any quantity of lines can be allowed to open up to the total number of lines, $j$ can range from one to the total number of lines (i.e. $j=1, \ldots,|\Lambda|$ ).

\section{TEST NETWORK RESUltS}

To test the formulation presented above, we used the well-known and widely-used IEEE 118-bus engineering test network. We modeled generator costs as linear, assumed resistance and shunt capacitance of lines were zero, ignored losses and reactive power, and did not perform any generator unit commitment, using instead the commitment profile of the test cases. ${ }^{5}$ The traditional DCOPF and modified optimal transmission switching DCOPF were tested on the case to assess potential benefits of optimal transmission switching. Also, this method was run on the system with various load profiles to highlight the benefits of system flexibility, and a contingency analysis was performed to assess effects on reliability.

\section{A. 118-bus model}

The IEEE 118-bus test case was used to test and analyze the optimal transmission switching formulation. Data for the test system was downloaded from University of Washington Power System Test Case Archive, and transmission line characteristics and generator variable costs were taken from the network as reported in [16]. This test model is based on a portion of the AEP network from 1962.

The system consists of 118 buses, 186 transmission lines, 19 committed generators with a total capacity of 5,859 MW, and 99 load buses with a total consumption of 4,519 MW. In

\footnotetext{
${ }^{5}$ In our model, we assume the generation unit commitment problem has already been solved. It is possible that an improved solution will result from simultaneously performing generator unit commitment and optimal transmission switching.
} 
what will be called the "base case", in which no transmission lines are opened, the system cost of meeting this consumption is $\$ 2,054 / \mathrm{h}$. This cost results from the DCOPF model with generator cost information from [16], where variable cost ranges from $\$ 0.19 / \mathrm{MWh}$ to $\$ 10 / \mathrm{MWh} .{ }^{6}$ Two lines out of the total of 186 are fully loaded, or congested.

First, we ran the MIP as presented above on the 118-bus model without constraint (6). This allowed the status of each line to be determined by the optimization, and for an unconstrained number of lines to open. While we encountered some difficulties in getting this problem to solve completely and to optimality (discussed below in Section V. ), the best found solution improved the system cost by 25 percent, or $\$ 511 / \mathrm{h}$, over the base case. In this solution, 38 lines are opened. There is no general trend in physical characteristics of the optimally-open lines; rather the decision to open a line or keep it closed depends on specific operating conditions, loads and generator costs.

To gain insight on how these lines are affecting system dispatch cost, we limit the number of open lines by including the GUB constraint. Allowing only one line to open $(j=1)$ results in $L_{1}=\{$ Line 153$\}$, meaning line 153 , connecting bus 89 to 91 , opens. ${ }^{7}$ Opening the line changes the power output of four generators. Generators at buses 25, 61, and 87 decrease output, and the generator at bus 111 increases output. As can be see in the table below, the increased generator is more expensive than two of the decreased generators, but the total cost savings, primarily driven by reducing output from the expensive generator at bus 87 , is significant. Power flow on transmission lines also change. The magnitude of power flow changes on 168 - or 90 percent - of lines, and the direction of power flow changes on four lines. The two lines that were constrained in the base case remain congested, but no additional lines become congested.

Repeating this analysis for $j=\{1, \ldots, 7\}$, we identified the sequence of subsets, $L_{j}$, and the objective function values for each of these topologies. For $\left|L_{j}\right| \leq\left|L_{j+1}\right|$ it is not necessarily true that $L_{j} \subset L_{j+1}$, see in particular the change from $j=6$ to $j=7$. In other words, these sequentially-found subsets are not necessarily subsets of one another. Also, for each additional open line, the system cost decreases, but at a decreasing rate (see TABLE II, below).

This implies two things about this network: one is that a small number of lines have a large impact on dispatch cost, and a large number of lines have a small impact. The other is that improving system cost by opening lines is not a linear process; serially opening the next line that provides the most

\footnotetext{
${ }^{6}$ These costs we used are on the order of 50 to 100 times smaller than typical generator costs. Realistic savings would be much higher than the $\$ 2,000 / \mathrm{h}$ found here. These costs were used because they are consistent with others found in the literature, and our goal was to remain as faithful to previous models as possible.

${ }^{7}$ This line forms a classic Wheatstone bridge along with lines connecting bus pairs $89 / 92,92 / 91,89 / 90$, and $90 / 91$. It has been shown that Wheatstone bridges can be associated with Braess Paradox, in which adding a line to a network can increase the cost of using that network [16] [24]. However, in our analysis not all optimally-open lines are the Wheatstone bridge link in such traditional network structures; for example the line from bus 4 to bus 5 is opened but is not the middle link of a Wheatstone bridge structure.
}

improvement will not necessarily produce the optimal network. Thus, to gain the majority of economic benefit from optimal transmission switching a large number of lines need not be switched, necessarily; however, the number of lines to be opened would need to be defined a priori in the optimization model to achieve the most benefit. Running the full optimization problem and then choosing only some of the lines to open would not be the best way to optimally operate the network. One possibility would be to define, in the optimization problem, a subset of lines eligible for switching based on reliability studies.

These conclusions have only been shown in the 118-bus network thus far, and analysis of other networks is needed before determining if they are general findings. However, if these findings are found to be true in other networks they may be useful in making the practice of optimal transmission switching feasible.

TABLE I

Changes In GENERATOR OUTPUT AFTER OPENING LINE 153

\begin{tabular}{ccc}
\hline \hline & $\begin{array}{c}\text { Variable Cost } \\
{[\$ / \mathrm{MWh}]}\end{array}$ & $\begin{array}{c}\text { Output Change } \\
{[\mathrm{MW}]}\end{array}$ \\
\hline Generator Bus & 0.434 & -5.27 \\
61 & 0.588 & -15.69 \\
87 & 7.142 & -32.97 \\
111 & 2.173 & 53.93 \\
\hline \hline
\end{tabular}

TABLE II

SEQUENCE OF LINE OPENINGS

\begin{tabular}{|c|c|c|c|}
\hline $\begin{array}{c}\text { Number of } \\
\text { Open Lines } \\
\text { Allowed } \\
(j) \\
\end{array}$ & $\begin{array}{c}\text { Open Lines } \\
\left(L_{j}\right) \\
\end{array}$ & $\begin{array}{c}\text { System } \\
\text { Dispatch } \\
\text { Cost } \\
\left(T C_{j}\right)\end{array}$ & $\begin{array}{c}\text { Percent } \\
\text { Savings in } \\
T C_{j}\end{array}$ \\
\hline 0 & - & $-\$ 2,054$ & - \\
\hline 1 & Line 153 & $-\$ 1,925$ & $6.3 \%$ \\
\hline 2 & Lines 132,153 & $-\$ 1,800$ & $12.4 \%$ \\
\hline 3 & Lines $132,136,153$ & $-\$ 1,646$ & $19.9 \%$ \\
\hline 4 & $\begin{array}{l}\text { Lines 132,136, } \\
153,162\end{array}$ & $-\$ 1,633$ & $20.5 \%$ \\
\hline 5 & $\begin{array}{l}\text { Lines } 64,132,136,153 \text {, } \\
162\end{array}$ & $-\$ 1,607$ & $21.8 \%$ \\
\hline 6 & $\begin{array}{l}\text { Lines } 64,69,132,136 \text {, } \\
153,162\end{array}$ & $-\$ 1,602$ & $22.0 \%$ \\
\hline 7 & $\begin{array}{l}\text { Lines } 64,86,132,136 \text {, } \\
146,153,161\end{array}$ & $-\$ 1,596$ & $22.3 \%$ \\
\hline $\begin{array}{l}\text { No restriction } \\
\text { (optimality not } \\
\text { proven yet) }\end{array}$ & & $-\$ 1,543$ & $24.9 \%$ \\
\hline
\end{tabular}

To ensure the large percent savings we saw was not due to the wide range of generator costs, we also ran the optimal transmission switching model with the three most expensive generator costs halved. This sensitivity analysis resulted in a 16.2 percent savings with ten lines removed. While this savings is smaller than that achieved with the original generator costs taken directly from the standard network model, it is still substantial and suggests that the high generator costs do not dominate the savings.

\section{B. Changing Load Profiles}

If opening certain lines make the solution more efficient, does this mean they should be opened permanently? Does the existence of alternative transmission topologies with improved dispatch cost mean the system was not optimally planned, that 
the transmission engineers designed a suboptimal system? Not necessarily. There are two reasons why a given transmission system may appear suboptimal. One is that in order to have a reliable network some lines that decrease the economic optimality may be necessary. Another reason is that different patterns of loading may benefit from different topologies; the optimal network for one generator/load scenario may be different from the optimal network of another.

To simulate different loading scenarios, we created two additional cases, called Peak and Off Peak, in which each load in the case above is scaled either up or down by ten percent, respectively. The load in the case presented above is referred to as the Shoulder case. Total load in the Peak case is 4,971 $\mathrm{MW}$, and in the Off Peak case is 4,067 MW, compared to total load of 4,519 MW in the Shoulder case.

For each of these two new cases, the MIP was performed with the GUB constraint, for $j=0, \ldots, 5$ and $j=0, \ldots, 4$. System cost decreased by 12.2 percent for the Peak load case for $j=5$, and by 17.8 percent for the Off-Peak case for $j=4$. The Peak load case is not feasible without opening at least one line. To analyze the Peak load case infeasibility, we increased transmission capacity and found a feasible solution with six overloaded lines, all of which have a capacity of $220 \mathrm{MW}$ and are overloaded by 112 to 149 percent. In the $j=1$ (feasible) case, three of these lines are loaded to capacity, and the others are within 1,7 and 20 percent of being fully loaded.

To gauge how much improvement comes from determining transmission topology based on actual system conditions, the optimal transmission topology of the Shoulder case was applied to the Peak and Off Peak load scenarios. The resulting system cost was compared to the optimal system cost determined by running the MIP on the specific load scenarios. In both the Peak and Off Peak cases, the optimal open lines are different from those optimally open in the Shoulder case, and the transmission topology that is best for the Shoulder load case results in a higher system dispatch cost, by two to eleven percent, compared to the optimal transmission topology determined for the Peak or Off Peak scenarios. These results are shown below in Fig. 1 and Fig. 2. In the Peak load case, opening the lines optimal to the Shoulder case results in a degradation of system cost from $j=2$ to $j=3$ and from $j=4$ to $j=5$. This occurs because the set of open lines was optimized for a different load profile.

TABLE III

PEAK LOAD REsults

\begin{tabular}{|c|c|c|c|c|c|}
\hline $\mathrm{j}$ & $\begin{array}{l}\text { Open } \\
\text { Lines }\end{array}$ & $\begin{array}{c}\text { System } \\
\text { Cost }\end{array}$ & $\begin{array}{c}\text { Open } \\
\text { Lines: } \\
\text { Shoulder } \\
\text { case } \\
\end{array}$ & $\begin{array}{c}\text { System } \\
\text { Cost }\end{array}$ & $\begin{array}{c}\text { Percent Diff. } \\
\text { in System } \\
\text { Cost }\end{array}$ \\
\hline 0 & & Infeas. & & Infeas. & \\
\hline 1 & 153 & $-\$ 2,738$ & 153 & $-\$ 2,738$ & $0 \%$ \\
\hline 2 & $\begin{array}{l}132,157 \\
132,153\end{array}$ & $-\$ 2,574$ & $\begin{array}{l}132,153 \\
132,136\end{array}$ & $-\$ 2,613$ & $2 \%$ \\
\hline 3 & & $-\$ 2,528$ & & $-\$ 2,677$ & $6 \%$ \\
\hline 4 & $\begin{array}{l}153,165 \\
78,132, \\
133,153, \\
165\end{array}$ & $-\$ 2,454$ & $\begin{array}{l}153,162 \\
64,132, \\
136,153, \\
162\end{array}$ & $-\$ 2,568$ & $11 \%$ \\
\hline
\end{tabular}

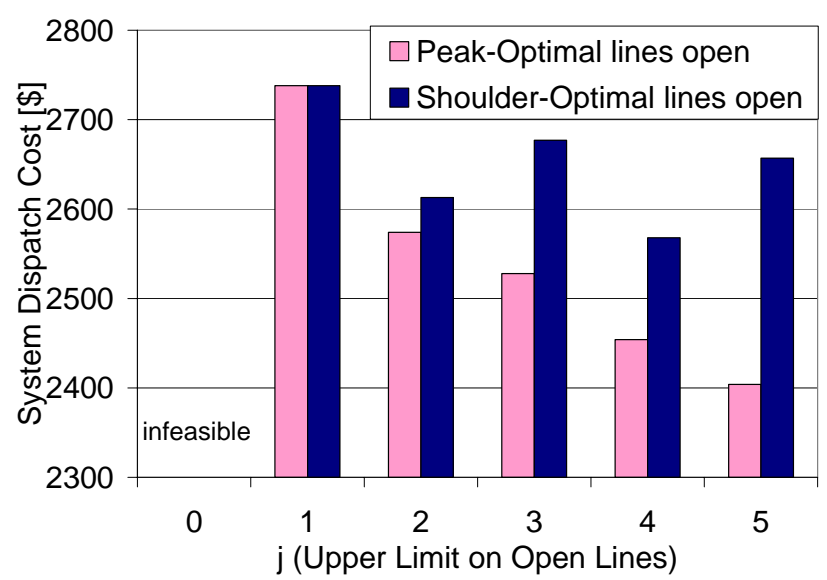

Fig. 1. Peak Load System Dispatch Cost

TABLE IV

OFF-PEAK LOAD RESULTS

\begin{tabular}{|c|c|c|c|c|c|}
\hline $\mathrm{j}$ & $\begin{array}{l}\text { Open } \\
\text { Lines }\end{array}$ & $\begin{array}{c}\text { System } \\
\text { Cost }\end{array}$ & $\begin{array}{c}\text { Open Lines: } \\
\text { Shoulder } \\
\text { case }\end{array}$ & $\begin{array}{c}\text { System } \\
\text { Cost }\end{array}$ & $\begin{array}{c}\text { Percent } \\
\text { Diff. in } \\
\text { System } \\
\text { Cost } \\
\end{array}$ \\
\hline 0 & & $-\$ 1,306$ & & $-\$ 1,306$ & $0 \%$ \\
\hline 1 & 132 & $-\$ 1,212$ & 153 & $-\$ 1,301$ & $7 \%$ \\
\hline 2 & $\begin{array}{l}132,136 \\
64,132,\end{array}$ & $-\$ 1,099$ & 132,153 & $-\$ 1,208$ & $10 \%$ \\
\hline 3 & $\begin{array}{l}136 \\
64,131,\end{array}$ & $-\$ 1,075$ & $\begin{array}{l}132,136,153 \\
132,136,\end{array}$ & $-\$ 1,097$ & $2 \%$ \\
\hline 4 & 132,133 & $-\$ 1,073$ & 153,162 & $-\$ 1,091$ & $2 \%$ \\
\hline
\end{tabular}

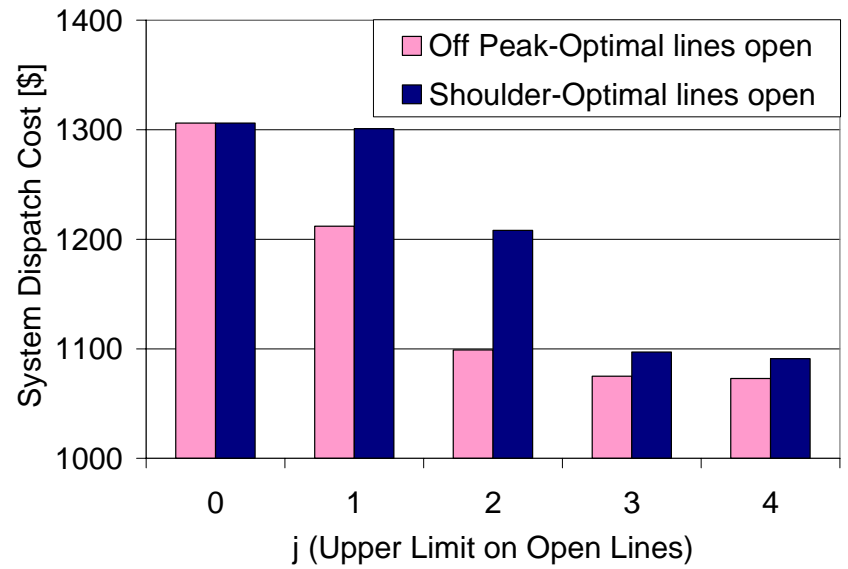

Fig. 2. Off-Peak Load System Dispatch Cost

These results indicate that a transmission network optimized for one particular pattern of load on a network is not necessarily optimal for another. Thus, allowing decisions about network topology to be made based on real-time system conditions (or daily or weekly forecasts) can result in a lowercost dispatch than using a static network optimized for a multi-period forecast.

\section{Contingency Analysis}

Reliability of the system paramount, and must be ensured by system operators. In this section we explore how the modified transmission networks, found via optimal transmission switching, perform under an $\mathrm{n}-1$ contingency 
analysis, in which it is determined whether the system can withstand the loss of one component or contingency.

To conduct the contingency analysis, the 118-bus test case (with Shoulder loads) was imported into PowerWorld Simulator 12. PowerWorld, rather than the GAMS model discussed above, was used for this analysis because it includes a well-tested contingency analysis tool, and using this existing tool was adequate and more convenient than developing one in GAMS. The authors are developing an n-1 DCOPF with optimal transmission switching to be used in future studies for more precise analysis of reliability issues, but for now, our goal was to ensure optimal transmission switching was not antithetical to maintaining security.

The PowerWorld $n-1$ contingency analysis consists of removing each specified contingency in turn and running a power flow, keeping generators at their previous output levels. If any additional generation is needed it is made up at the slack bus. After re-calculating the power flows, all violations of line capacity, bus voltages and islanded load are reported for that specific contingency. This is repeated for all identified contingencies. In our analysis we defined all transmission lines as contingencies, and a violation was considered any line flow above 110 percent of normal, a value that is conservative compared to acceptable post-contingency line flow limits. ${ }^{8}$

First, an $n-1$ contingency analysis was performed for the base case transmission topology. Twenty nine line violations were found in the base case, with line overloads ranging from 110 to 237 percent. $^{9}$

Next, we opened line 153, the single line that improves the system dispatch cost the most, and conducted the $\mathrm{n}-1$ contingency analysis. This resulted in 28 violations: 23 violations that occurred for the same contingency and on the same line as for the base case, and five violations not occurring in the base case. Six violations that occurred in the base case did not appear for the new topology. Of the 23 shared violations, 13 of the line overloads were ameliorated (although not removed), eight lines were loaded at a higher level, and two remained unchanged in magnitude.

We continued opening sets of lines, identified above in TABLE II, and running the contingency analysis, up to $j=5$. All of the modified topologies resulted in some violations in the $\mathrm{n}-1$ contingency analysis, but this is not surprising because the base case itself had violations. All modified topologies shared at least 20 violations with the base case, and all improved some of these shared violations. All topologies resulted in new violations, but also relieved other violations that existed in the base case. These results are summarized in TABLE V, where the number of total violations is listed for each network configuration along with the break-down of how

\footnotetext{
${ }^{8}$ For example, in PJM emergency (i.e. single contingency) limits are generally 111 to 117 percent of normal, and if there are multiple contingencies, the acceptable "load dump" limit is 115 percent of the emergency limit [25] .

${ }^{9}$ Islanded load is also reported, but in this system all but one occurrence of islanded load was a result of a radial load. Radial loads are not required to survive contingencies, according to the ERO Reliability standards adopted by FERC in Order 696, and thus were ignored in this analysis [26] (See standard TPL-002)
}

many of these violations existed in the base case, how many did not, and how many base case violations did not exist under the optimal modified network topology.

TABLE V

VIOLATIONS RESULTING FROM N-1 CONTINGENCY ANALYSIS

\begin{tabular}{ccccc}
\hline \hline \multicolumn{5}{c}{ VIOLATIONS RESULTING FROM N-1 CONTINGENCY ANALYSIS } \\
\hline$j$ & Violations & In Base Case & $\begin{array}{c}\text { Not in Base } \\
\text { Case }\end{array}$ & $\begin{array}{c}\text { Base Case } \\
\text { Alleviated }\end{array}$ \\
\hline 0 & 29 & - & - & - \\
1 & 28 & 23 & 5 & 6 \\
2 & 34 & 25 & 9 & 4 \\
3 & 43 & 22 & 21 & 7 \\
4 & 40 & 20 & 20 & 9 \\
5 & 44 & 20 & 24 & 9 \\
\hline \hline
\end{tabular}

Results from this contingency analysis indicate that operating a system with optimal transmission switching can have a mixed effect on system reliability. Changes that improve economic operation make some improvements to system security, by alleviating or completely removing previously-existing violations, but also may weaken reliability in the absence of operational procedures to prevent it. Thus, optimal transmission switching does not intrinsically cause reliability problems, and is worthy of further analysis.

An important part of system reliability, whether in a static network or a controllable one, is generation dispatch. System operators already have tools to ensure reliable operation of transmission systems, including generation dispatch. Security constrained optimal power flow (SCOPF) models are used to dispatch systems reliably, and there is no reason these would not be compatible with the optimal transmission switching DCOPF presented here. In other words, changing the transmission network in a reliable way may be accomplished by repurposing the same tools used to ensure reliability of generator dispatch today. By combining existing tools for reliable dispatch of generation with optimal transmission switching, it is likely possible operators could maintain a reliable system while reducing system cost. Preliminary results from the DC SCOPF with optimal transmission switching being developed by the authors result in savings of 15.5 percent for the IEEE 118-bus network; additional work is needed to discuss these results in more detail.

\section{Practical Solution Difficulties}

Computational issues are central to the practicality of implementing optimal transmission switching. The MIP must be able to solve on market or control area scale systems in the time frame required by the system operator. In this section we discuss our solution process and some problems we faced along with potential solutions. While other methods, such as Benders decomposition [5], or heuristics, like genetic algorithms [6], may be helpful in solving this problem, we focus on solving the MIP formulation directly since it has been identified as a promising direction for production-scale networks [17].

Although the 118-bus network is small compared to realworld networks, it is very large in terms of potential solutions, and thus computationally difficult to solve. There are $2^{186}$ potential transmission network topologies, far too many for traditional enumeration techniques. The standard version of 
CPLEX was unable to solve this problem to full optimality. Instead, we used a partitioning and parallel solution approach, described in the appendix. This means the problem was separated into a collection of subproblems in which the domains of the binary variables did not overlap, and these subproblems were then solved simultaneously. Each of the partitioned problems was solved using CPLEX, with a variety of non-default settings for its algorithmic options. CPLEX is a sophisticated implementation of a branch-and-cut / branchand-bound hybrid method for solving MIPs, which is described in the appendix of this paper.

To gain insight into the problem, we used the GUB constraint, discussed above, to limit the number of lines to be taken out. The following table shows the result of these exercises; all problems were solved to optimality. Larger values of $j$ were deemed impractical for this solution technique because the solution times appeared to be growing exponentially. ${ }^{10}$

TABLE VI

SOLUTION AND COMPUTATION TIME FOR OPTIMAL TRANSMISSION SWITCHING FOR $J$ OR FEWER LINES REMOVED

\begin{tabular}{|c|c|c|}
\hline$j$ & Objective Function & $\begin{array}{l}\text { Time to solve to optimality } \\
\text { (hr:min:sec) }\end{array}$ \\
\hline 6 & -1602.0559 & $00: 06: 57$ \\
\hline 7 & -1595.8763 & $00: 16: 40$ \\
\hline 8 & -1568.0766 & $00: 41: 20$ \\
\hline 9 & -1557.0102 & $02: 00: 10$ \\
\hline 10 & -1553.9278 & 05:04:00 \\
\hline 11 & -1553.4292 & $02: 24: 00$ \\
\hline 12 & -1549.6355 & $41: 37: 00$ \\
\hline
\end{tabular}

Using the solution for $j=12$ as a feasible starting point, various techniques (outlined in the appendix) were used to create and solve subproblems in which certain binary variables were fixed to zero or one. These subproblems were solved either to optimality (at which point the solutions with suboptimal objective functions were discarded) or split into more subproblems in an attempt to find the optimal solution of the original problem. From this process, one outstanding subproblem was not solved to optimality before reaching a node limit. ${ }^{11}$

The best found objective function value is -1542.8904 , with a known upper bound of -1542.8900 . This translates to an optimality gap of $3 \times 10^{-7}$. It is possible that this is optimal, and that we have found the optimal solution and transmission network. The problem is proving optimality. It may be possible that further computation could lower the upper bound, however it may not. If this gap is caused by the small rounding errors introduced by cuts added to the constraint space by the solution technique we will never reach a zero gap. It has been observed that these cuts can be almost linearly dependent on one another, and hence can lead to numerical difficulties with rounding error. Regrettably, the addition of

\footnotetext{
${ }^{10}$ The problem with $\mathrm{j}=13$ was terminated suboptimally after 40 hours.

${ }^{11}$ As an indication of how large this problem is, the outstanding problem is the $359,422^{\text {nd }}$ subproblem of the original partition, and the $958^{\text {th }}$ partition of this subproblem.
}

cuts to the model is imperative to reduce the amount of nodes that the branch and bound process has to explore.

Much of the computational difficulty comes from proving optimality of the solution. While finding the optimal solution and proving optimality is an intriguing theoretical and academic question, it appears that the majority of benefits from optimal transmission switching can be found quickly, by switching a few lines. As a practical matter we may not need to prove optimality to enjoy substantial gains.

\section{PRACTICAL IMPLICATIONS}

Before implementing optimal transmission switching, a variety of practical considerations would have to be satisfied.

First and foremost, maintaining reliability would remain the primary goal. Switching lines in and out must be done in a way that is compatible with system security. Current practice in many control areas indicates that this is not only possible but being done. What is proposed in this paper is a way to automate decisions about opening or closing lines in order to make more efficient and transparent decisions. The optimization formulation presented here would need to be modified to include constraints preventing insecure networks and dispatches, or be combined with security and transient analysis and $\mathrm{AC}$ power flow studies.

For optimal transmission switching to work, communication and switching equipment must be installed and confirmed to be reliable, so lines can be opened and closed quickly and under full understanding of the system conditions. This switching technology exists, thus implementing optimal transmission switching would be a matter of investing in available technologies and training operators and technicians, not developing new technologies. Limits on equipment, such as how often or how quickly lines can be switched in and out, can be easily added to the optimization formulation as constraints. Given the potentially large cost savings identified here this capital and personnel outlay for this 'smart grid' technology may be justified.

Another consideration is that while the solution time to prove optimality for these problems is long, it takes a fraction of this time to arrive at a solution with most of the savings. An analysis of who gains and who bears potential costs would provide more insight into the question of whether it is politically feasibility to operate a system in a way that provides large benefits but may not be proven optimal. In addition, there is a great potential for improving solution techniques. Just as system operators use their specific knowledge of the network to make determinations about appropriate and desirable actions to take, particular knowledge of the specific system will aid in development of better solution heuristics. In addition, improvements in computing power and techniques will aid in solving of large problems [18] [19].

The transmission system is typically funded by regulated rates, even in areas that have opened generation to competition. Thus switching lines in and out does not have the same financial implications as committing or de-committing generators owned by private companies, because transmission lines are not paid for hours operated or power transferred. The switching decisions, however, would need to be made by 
impartial organizations having no financial interest in the effects of changing the system topology. For instance, a vertically-integrated utility with control over switching lines may be able to modify the network to favor their own generators or discriminate against competitors. This would be a situation to guard against.

Also, financial transmission rights need to be considered in a non-static network. Revenue adequacy for transmission rights is not a new phenomenon. Insufficiently funded transmission rights have been found to occur when transmission lines have maintenance or forced outages, when new transmission lines are built, even when the model used to calculate the rights uses DC approximations [20] [21] [22] [23]. The introduction of OTS may affect some aspects of this issue, but not the essence of the problem. Appropriate handling of FTRs, as well as other financial and market issues, need further study before optimal transmission switching could be implemented.

Before implementation, it is also necessary to determine whether the benefits of implementing optimal transmission switching would outweigh the costs. There are potentially many costs, including investing in or upgrading switching and communications equipment, developing computer software, and training personnel. While this study is not meant to be a formal cost/benefit analysis, we conclude that the potential benefits are promising enough to warrant further study of this optimal transmission switching.

\section{CONCLUSION}

This paper presents an optimization model for determining optimal transmission network topology and generation output to meet a static load. We found that large improvements in system dispatch cost can be achieved by optimizing the transmission network, switching lines in and out, based on system conditions. An improvement in system cost of 25 percent was achieved in the IEEE 118-bus test network. Most of the improvement in dispatch cost is realized by changing the status of a few lines and takes a short time to compute. Proving optimality can take a very long time, involve complex solution techniques, and can involve opening many lines.

Switching transmission lines to improve economic dispatch does not necessarily have a negative impact on reliability, according to the contingency analysis performed in this paper. It would be necessary to perform contingency analyses on any particular system using controllable networks, as they are performed on static networks, to ensure generation dispatch is secure and reliability is not negatively impacted.

Real-time (or close to real-time) control can result in more efficient transmission topologies than static ones, even if the static ones were originally designed to be optimal. The analysis presented here could also aid in finding better investment opportunities.

This paper is a first step in analyzing the potential benefits of optimal transmission switching. More research, particularly in the areas of reliability and cost or surplus allocation, will provide more insight into the possibilities for controllable, more flexible networks to be part of the solution for meeting growing demand in a transmission-constrained world.

\section{APPENDIX}

Large-scale implementation of MIPs depends on developing smart solution techniques. For a problem of any realistic size, brute-force, branch-and-bound methods are not practical. Thus, the practical implementation of optimal transmission switching includes understanding computational solvers and developing heuristics that can improve solution time. This appendix briefly describes the techniques CPLEX uses to solve MIP problems, and then reports on the solution techniques and heuristics we employed to solve the optimal transmission switching problem for the 118-bus test case.

\section{A. CPLEX MIP Solution Technique}

CPLEX is a sophisticated implementation of a branchand-cut / branch-and-bound hybrid method for solving MIPs. To commence a solution, CPLEX solves the linear programming relaxation of the MIP, where the binary variables are relaxed and allowed to take on any value in the interval $[0,1]$. This subproblem forms the root of the branchand-bound tree and can be solved by any linear programming algorithm. To speed up the solution of this initial problem, logical implications are used to "preprocess" the problem, a method that fixes variables at their optimal values and removes redundant constraints by analyzing the objective function and particular form of the constraints.

If this root relaxation results in all binary variables having binary values, the solution is optimal for the original problem and the process terminates. Otherwise, the value of the objective function of the relaxed problem becomes an upper bound on the optimal solution of the maximization MIP, and a cutting plane algorithm is used to find linear constraints that are satisfied by all feasible integer points, but violated by the current fractional solution. ${ }^{12}$ If such an inequality is found, this "cut" is added to the linear program. Adding a cut always decreases the optimal value of a maximization linear program, the feasible region is always reduced, so the cut will either decrease or leave unchanged the value of the upper bound. Cuts are repeatedly added until an optimal integer solution is found, or until a limit is reached on the number of cuts that can be generated, or it becomes impossible or too (computationally) expensive to find another cutting plane.

An integer feasible point gives a lower bound on the optimal objective value. The optimality gap is the difference between the lower and upper bounds. The CPLEX solver will terminate if the optimality gap becomes suitably small. If not, a variety of heuristics are used to try to generate an integer feasible point. Sometimes good feasible solutions or even optimal solutions can be generated by such heuristics quickly; otherwise the branch and bound phase of the algorithm is started.

The branch and bound algorithm generates a binary search tree whose nodes correspond to linear programming subproblems. The tree is rooted at the relaxation subproblem

\footnotetext{
${ }^{12}$ See [27] for more on cutting planes and techniques for finding these linear constraints.
} 
augmented by the additional cuts. The branching process from a given node identifies a binary variable that has a fractional value in the corresponding subproblem solution and splits the node into two nodal subproblems: one of which has an additional restriction that the binary variable is zero, and the other has the restriction that it is one. The fractional variable for branching is chosen via a node selection rule and CPLEX encodes a variety of techniques to make this choice. Thus branching generates new nodes in the search tree.

At any given stage there are nodes in the tree that have been generated but not yet solved. A variable selection rule is used to determine which node to process next. The corresponding subproblem is solved, and one of three things can happen. First, the subproblem is infeasible, or its objective value is poorer than a known integer feasible point. In this case, the node is fathomed (i.e. it is discarded since it can only produce integer solutions where the objective value is worse than the known achievable lower bound). One possible result is that a feasible integer point is found from solving the subproblem that is better than the best known integer feasible point. In this case, the lower bound is updated (and the solution stored), and the node is again fathomed. The other possibility is that some binary variables have fractional values, and in this case the node is split using the branching rule outlined above. During the branch and bound process, more cutting planes can be generated and added to the subproblems.

\section{B. Technique used in this paper}

Although the problem examined in this paper is small compared to real-world networks, it is very large in terms of potential solutions, and the standard version of CPLEX was unable to solve this problem. Instead, to solve it, a partitioning and parallel solution approach was used. This means we separated the problem into a collection of subproblems, in which the domains of the discrete variables did not overlap, and solved each of the subproblems simultaneously. Each of the partitioned problems was solved using the CPLEX code, with a variety of non-default settings for its algorithmic options.

First, a four-threaded version of CPLEX was used to determine a good solution, with objective value -1542.8904 . This solution provided a cutoff value of -1543 : no solution below this value is of interest, thus any node in the branch and bound tree with a solution less than this is deleted.

Next, we fixed a subset of variables (based on examining solutions from the problem including the GUB constraint, as mentioned above in Section V. ) to see if certain subsets have large impacts on the solution. We found that a subset of 19 of the lines seemed to generate subproblems that were relatively easy to fathom in the branch and bound process; in other words for many settings of these variables, the corresponding objective function values were quickly shown to be provably worse than the best known solution. We used this set of 19 variables, and fixed their values at all combinations of 0 or 1 , thereby partitioning the solution space into $2^{19}=524,288$ subproblems. Although choosing the 19 variables was carried out in an ad-hoc manner, it may be useful to use domainspecific information to determine subsets of the fixed lines; we have begun to examine the benefits of this and preliminary results look promising. We attempted to solve each of these problems in parallel, but with a slight change to the default options of CPLEX. In particular, we ran CPLEX on default options for a short period $(<10$ seconds $)$ in order to get a reasonably good initial solution, and then interrupted the process to set an objective function minimum (a cutoff value) of -1543 and an upper bound on the number of nodes (using CPLEX function "nodelim") of 10,000. This non-standard setting, which typically generates a fairly poor incumbent solution in the first period when the default CPLEX options are in effect, seems to substantially improve the overall performance of CPLEX (i.e. the existence of a solution when the cutoff is applied is extremely helpful to the solver). Running these subproblems, all but 141 resulted in a computational proof that the objective function was smaller than -1543 (our known cutoff value) in this subproblem and thus were eliminated from consideration. Note that only the 141 remaining subproblems encountered the node limit - all the other subproblems were fathomed before the number of nodes reached the limit.

For these remaining 141 subproblems, we attempted to solve the problems again using the above technique but with a time limit of 20 seconds in the first run, and a node limit of 100,000 in the second run. After this, a collection of 80 subproblems were left. These values were chosen somewhat arbitrarily, based on experience with other MIP problems and some trial and error.

For each of these 80 subproblems, a further partition was generated, namely taking another 10 variables and fixing their values at zero and one. This resulted in 80x1024 subproblems to process: 81,920 MIP models to solve using CPLEX. The option settings were kept the same, except the first run had a time limit of 5 seconds, and the second run was allowed 100,000 nodes. Again, these values were chosen heuristically, and could all have been set to the same numbers as above.

Ninety-seven problems remained unfathomed in this collection after this set of CPEX runs. Many of the 81,920 subproblems were processed quickly, but the remaining subproblems all were unable to prove nonoptimalilty after exploring 100,000 nodes. These problems were each further partitioned into 4 subproblems. New options were used for CPLEX (cutsfactor 10, repeatpresolve 3, probe 3), resulting in a collection of 17 remaining subproblems. A further refinement into 4 subproblems for each of these 17 resulted in 14 outstanding subproblems. One more refinement into four subproblems left two outstanding subproblems. One of these was fathomed with two more refinements, and one subproblem remains. It is clear that the partitioning process quickly identifies the optimal value of a subset of the variables that contain good solutions, but even with these values set there remains a large numbers of choices to be made.

The outstanding problem is indexed as "359422.958" indicating that it was the $359,422^{\text {nd }}$ subproblem of the original partition of 524,288 problems, and the $958^{\text {th }}$ partition of this subproblem (out of 1024). The indices are used in a systematic manner to determine which variables get set to zero and which to one.

As mentioned above, the result of these computations is a best found value of -1542.8904 , and an upper bound of 
-1542.8900 . It may not be possible to close the remaining optimality gap. The remaining problem (that is a partition of the original problem with a collection of the binary values fixed) contains a solution of value -1542.8909 , which would mean that it is indeed suboptimal and the best found solution above is optimal. However, it is possible that rounding errors from the cutting plane procedure may prevent us from reliably fathoming this outstanding node.

\section{ACKNOWLEDGMENT}

The authors gratefully acknowledge Alper Atamturk of UC Berkeley, Karla Hoffman of George Mason University, and Alex Meeraus of GAMS Corporation for their assistance on the problem described in this paper. We would also like to thank Arne Stolbjerg Drud at ARKI Consulting and Development A/S for help with the MIP formulation, in particular with the modification of Kirchhoff's laws constraints. Also, we would like to acknowledge Mary Cain and Eric Hseih for their help, and offer a special thanks to Kory Hedman for his assistance. The authors take full responsibility for the contents of the paper.

\section{REFERENCES}

[1] Northeast Power Coordinating Council, "Guidelines for Inter-Area Voltage Control," November. 1997.

[2] ISO-NE, "ISO new england operating procedure no. 19: Transmission operations," April 13, 2007. pp 7-8

[3] F. D. Galiana, D. T. McGillis and M. A. Marin, "Expert systems in transmission planning," Proceedings of the IEEE, vol. 80, pp. 712-726, 1992.

[4] J. Choi, T. D. Mount, R. J. Thomas and R. Billinton, "Probabilistic reliability criterion for planning transmission system expansions," IEE Proc., Gener. Transm. Distrib., vol. 153, pp. 719-727, November. 2006.

[5] S. Binato, M. V. F. Pereira and S. Granville, "A new Benders decomposition approach to solve power transmission network design problems," Power Systems, IEEE Transactions on, vol. 16, pp. 235-240, 2001.

[6] G. Granelli, M. Montagna, F. Zanellini, P. Bresesti, R. Vailati and M. Innorta, "Optimal network reconfiguration for congestion management by deterministic and genetic algorithms," Electr. Power Syst. Res., vol. 76, pp. 549-556, APR. 2006.

[7] G. Schnyder and H. Glavitsch, "Security Enhancement using an Optimal Switching Power Flow," IEEE Trans. Power Syst., vol. 5, pp. 674-681, MAY. 1990.

[8] G. Schnyder and H. Glavitsch, "Integrated Security Control using an Optimal Power Flow and Switching Concepts," IEEE Trans. Power Syst., vol. 3, pp. 782-790, MAY. 1988.

[9] R. Bacher and H. Glavitsch, "Network Topology Optimization with Security Constraints," IEEE Trans. Power Syst., vol. 1, pp. 103-111, NOV. 1986.

[10] J. G. Rolim and L. J. B. Machado, "A study of the use of corrective switching in transmission systems," IEEE Trans. Power Syst., vol. 14, pp. 336-341, FEB. 1999.
[11] W. Shao and V. Vittal, "Corrective switching algorithm for relieving overloads and voltage violations," IEEE Trans. Power Syst., vol. 20, pp. 1877-1885, NOV. 2005.

[12] R. P. O'Neill, R. Baldick, U. Helman, M. H. Rothkopf and W. Stewart, "Dispatchable transmission in RTO markets," IEEE Trans. Power Syst., vol. 20, pp. 171-179, FEB. 2005.

[13] S. M. Harvey, W. W. Hogan and S. L. Pope, "Transmission Capacity Reservations and Transmission Congestion Contracts," June 6, 1996. 1996.

[14] H. Chao and S. Peck, "A Market Mechanism for Electric Power Transmission," Journal of Regulatory Economics, vol. 10, pp. 25-59, July. 1996.

[15] R. Baldick, Applied Optimization: Formulation and Algorithms for Engineering Systems. Cambridge University Press, 2006,

[16] S. A. Blumsack, "Network Topologies and Transmission Investment Under Electric-Industry Restructuring," pp. 1-283, May. 2006.

[17] D. Sun, "Challenges for market applications in large RTO markets," in Power Engineering Society General Meeting, 2005. IEEE, 2005, pp. 28562858 Vol. 3

[18] R. E. Bixby, "Solving real-world linear programs: A decade and more of progress," Oper. Res., vol. 50, pp. 3-15, JAN-FEB. 2002.

[19] Bussieck, M., Ferris, M. C., Meeraus, A. "Grid Enabled Optimization with GAMS." Technical Report, Computer Sciences Department, University of Wisconsin. 2007.

[20] W. W. Hogan, "Financial Transmission Rights Formulation," pp. 1-57, March 31, 2002. 2002.

[21] T. Kristiansen and J. Rosellón, "A Merchant Mechanism for Electricity Transmission Expansion," JRE, vol. 29, pp. 167-193, March. 2006.

[22] B. C. Lesieutre and I. A. Hiskens, "Convexity of the set of feasible injections and revenue adequacy in FTR markets," Power Systems, IEEE Transactions on, vol. 20, pp. 1790-1798, 2005.

[23] M. H. Liu and G. Gross, "Role of distribution factors in congestion revenue rights applications," IEEE Trans. Power Syst., vol. 19, pp. 802-810, MAY. 2004

[24] I. Milchtaich, "Network Topology and the Efficiency of Equilibrium," Games and Economic Behavior, vol. 57, pp. 321-346, 2006.

[25] PJM, “PJM Manual 03: Transmission Operations," May 24, 2007. Available at http://www.pjm.com/contributions/pjm-manuals/pdf/m03.pdf

[26] U.S. Federal Energy Regulatory Commission, "Mandatory Reliability Standards for the Bulk-Power System," Order no. 693, 72 FR 16416, April 4, 2007.

[27] G. Nemhauser and L. Wolsey. Integer and Combinatorial Optimization, New York: John Wiley and Sons, 1988. 